\title{
A model intercomparison analysing the link between column ozone and geopotential height anomalies in January
}

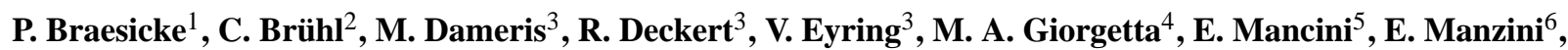 \\ G. Pitari ${ }^{5}$, J. A. Pyle ${ }^{1}$, and B. Steil ${ }^{2}$ \\ ${ }^{1}$ NCAS-Climate, Department of Chemistry, University of Cambridge, Cambridge, UK \\ ${ }^{2}$ Max-Planck-Institut für Chemie, Mainz, Germany \\ ${ }^{3}$ DLR Oberpfaffenhofen, Institut für Physik der Atmosphäre, Wessling, Germany \\ ${ }^{4}$ Max-Planck-Institut für Meteorologie, Hamburg, Germany \\ ${ }^{5}$ University of L'Aquila, L'Aquila, Italy \\ ${ }^{6}$ Istituto Nazionale di Geofisica e Vulcanologia e Centro Euro-Mediterraneo per i Cambiamenti Climatici, Bologna, Italy
}

Received: 27 August 2007 - Published in Atmos. Chem. Phys. Discuss.: 2 November 2007

Revised: 28 February 2008 - Accepted: 23 April 2008 - Published: 9 May 2008

\begin{abstract}
A statistical framework to evaluate the performance of chemistry-climate models with respect to the interaction between meteorology and column ozone during northern hemisphere mid-winter, in particularly January, is used. Different statistical diagnostics from four chemistry-climate models (E39C, ME4C, UMUCAM, ULAQ) are compared with the ERA-40 re-analysis. First, we analyse vertical coherence in geopotential height anomalies as described by linear correlations between two different pressure levels (30 and $200 \mathrm{hPa}$ ) of the atmosphere. In addition, linear correlations between column ozone and geopotential height anomalies at $200 \mathrm{hPa}$ are discussed to motivate a simple picture of the meteorological impacts on column ozone on interannual timescales. Secondly, we discuss characteristic spatial structures in geopotential height and column ozone anomalies as given by their first two empirical orthogonal functions. Finally, we describe the covariance patterns between reconstructed anomalies of geopotential height and column ozone. In general we find good agreement between the models with higher horizontal resolution (E39C, ME4C, UMUCAM) and ERA-40. The Pacific-North American (PNA) pattern emerges as a useful qualitative benchmark for the model performance. Models with higher horizontal resolution and high upper boundary (ME4C and UMUCAM) show good agreement with the PNA tripole derived from ERA-40 data, including the column ozone modulation over the Pacfic sector. The model with lowest horizontal resolution does not
\end{abstract}

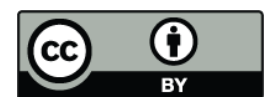

Correspondence to: P. Braesicke (peter.braesicke@atm.ch.cam.ac.uk) show a classic PNA pattern (ULAQ), and the model with the lowest upper boundary (E39C) does not capture the PNA related column ozone variations over the Pacific sector. Those discrepancies have to be taken into account when providing confidence intervals for climate change integrations.

\section{Introduction}

To understand chemistry-climate interactions we have to understand the intricate coupling between meteorology and ozone. Here, we will focus on the period 1980-1999, assessing the ability of chemistry-climate models (CCMs) to reproduce the observed interannual variability in monthly mean fields on selected pressure levels in the northern hemisphere during mid-winter, in particular January. This period is crucial for setting up the spring dilution of ozone and therefore the dynamical ozone trend in spring (e.g. Braesicke and Pyle, 2003). In addition, chemical ozone loss at the vortex edge will start during January and the overall relationship between the volume of low temperatures (below around 195 K) and ozone loss over the winter will start to develop (Rex et al., 2004). The ozone trend resulting from dilution and chemical loss is an important quantity in the context of policy making, which needs to be informed by modelling of the future development of the ozone layer.

Here, we use a form of model evaluation which attempts to identify processes and their linkages (e.g. Eyring et al., 2005; as compared to a classical climatological approach, e.g. Randel et al., 2004) looking at links between ozone and meteorology. There are many ways to reveal those linkages in

Published by Copernicus Publications on behalf of the European Geosciences Union. 


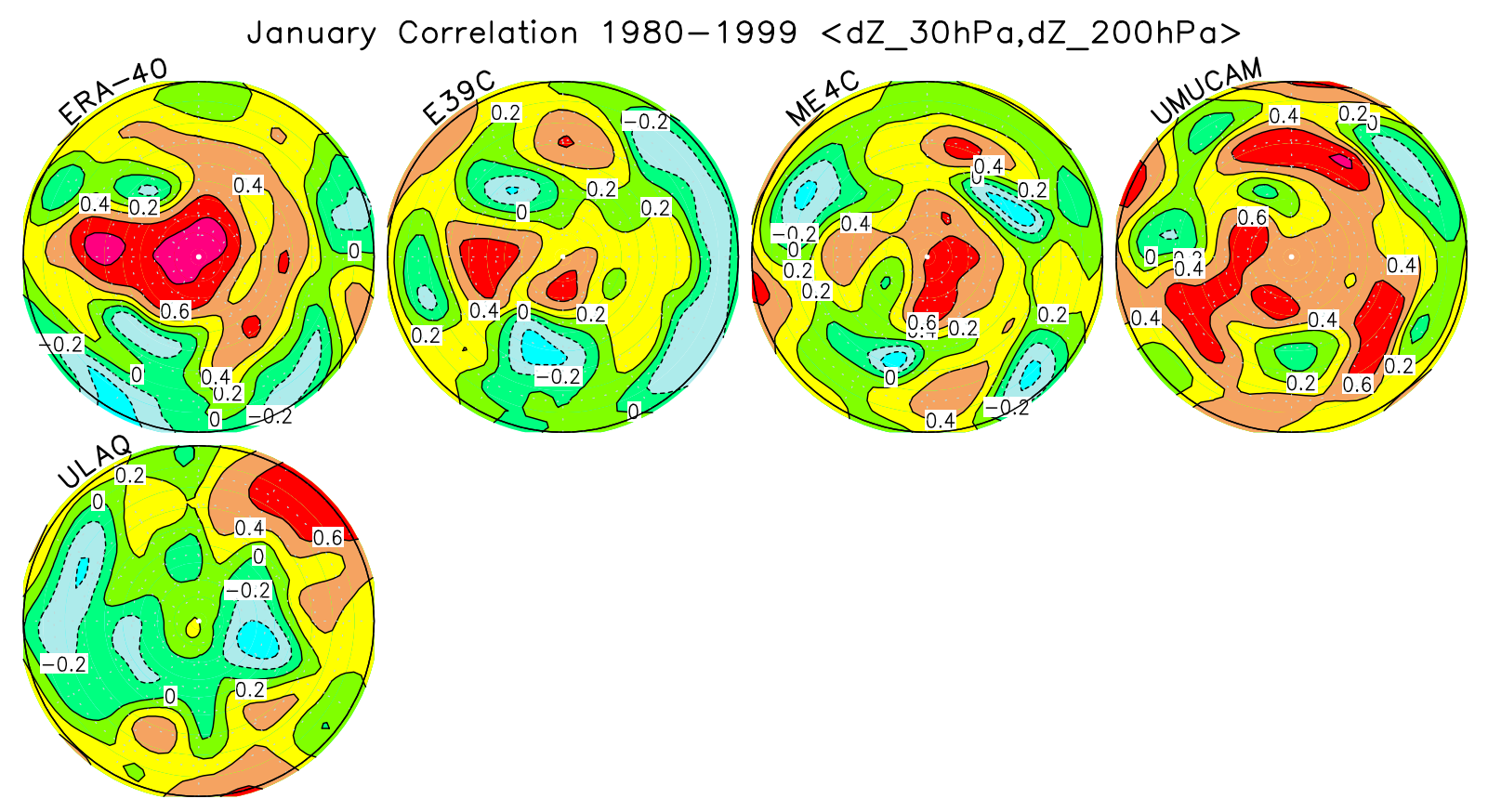

Fig. 1. Correlation between January monthly mean geopotential height anomalies at 200 and $30 \mathrm{hPa}$ during the time period $1980-1999$ in the northern hemisphere. Absolute values larger 0.44 can be considered to be significant at the $95 \%$ confidence level. The Greenwich meridian is at $6 \mathrm{o}^{\prime}$ clock and the southernmost latitude is at $20^{\circ} \mathrm{N}$.

idealised model experiments, but quite often the experimental design is necessarily guided by the needs of assessments and not by our aim to understand the working of our models. Many additional sensitivity studies are often not possible due to time and computational constraints. We are aiming to use existing "scenario"/"typical climate" runs of models and to compare them within a unified statistical framework, diagnosing local correlations/covariances to look at the link between column ozone and meteorology in terms of interannual variability on the northern hemisphere during midwinter. There are two levels of insight we can gain from this exercise: How does the coupling between meteorology and column ozone work in a single model? How do the models and a "proxy of observation" (re-analysis data) compare to each other? What can we learn about the coupling by looking at the discrepancies?

The use of monthly mean data, the pre-selection of month (January) and pressure levels (mostly $200 \mathrm{hPa}$ and $30 \mathrm{hPa}$ ) used in this analysis are largely guided by the experience gained in the validation and use of the Met Offices Unified Model (UM) with parameterised stratospheric chemistry (UMUCAM, e.g. Braesicke and Pyle, 2003). The $200 \mathrm{hPa}$ level is the lowest upper tropospheric level in which significant zonal mean changes in ozone and heat flux changes are just detectable in idealised 20 year climate change experiments in the UMUCAM (see e.g. Figs. $2 b$ and 6 in Braesicke and Pyle, 2004). In addition Braesicke et al. (2003) established a robust relation between $200 \mathrm{hPa}$ geopotential heights and column ozone in UMUCAM and the SLIMCAT CTM column ozone driven by ECMWF analysis for January in the Atlantic/European sector. The impact of vortex strength on high latitude column ozone in UMUCAM during January is strong and is a precondition for spring ozone anomalies in middle latitudes (Braesicke and Pyle, 2003). Even though the initial motivation for choosing the month and levels are largely based on UMUCAM, there is no evidence that this choice disadvantages one of the other participating models. In addition, for the data sets used the separation of the associated Eigenvalues (discriminable and strictly monotonic decreasing between empirical orthogonal functions (EOFs) 1,2 and 3 in a singular value decomposition sense) is monitored to assure the separation, correct order and linear independence of the EOFs. Compared to other winter months this separation is best in January, the month for which we will present our analysis.

A small number of different underlying mechanisms determine the correlation (covariance) patterns between geopotential height anomalies at $200 \mathrm{hPa}$ and column ozone for different latitude regimes. In middle latitudes we expect a strong modulation of column ozone by the height of the tropopause, which in our case is approximated using geopotential height anomalies at $200 \mathrm{hPa}$. A high/low tropopause will relate to low/high column ozone and will therefore lead to a negative correlation (e.g. Dobson, 1930; Orsolini et al., 1998 and Steinbrecht et al., 1998). In high latitudes we expect the reverse. Negative/positive geopotential height anomalies 


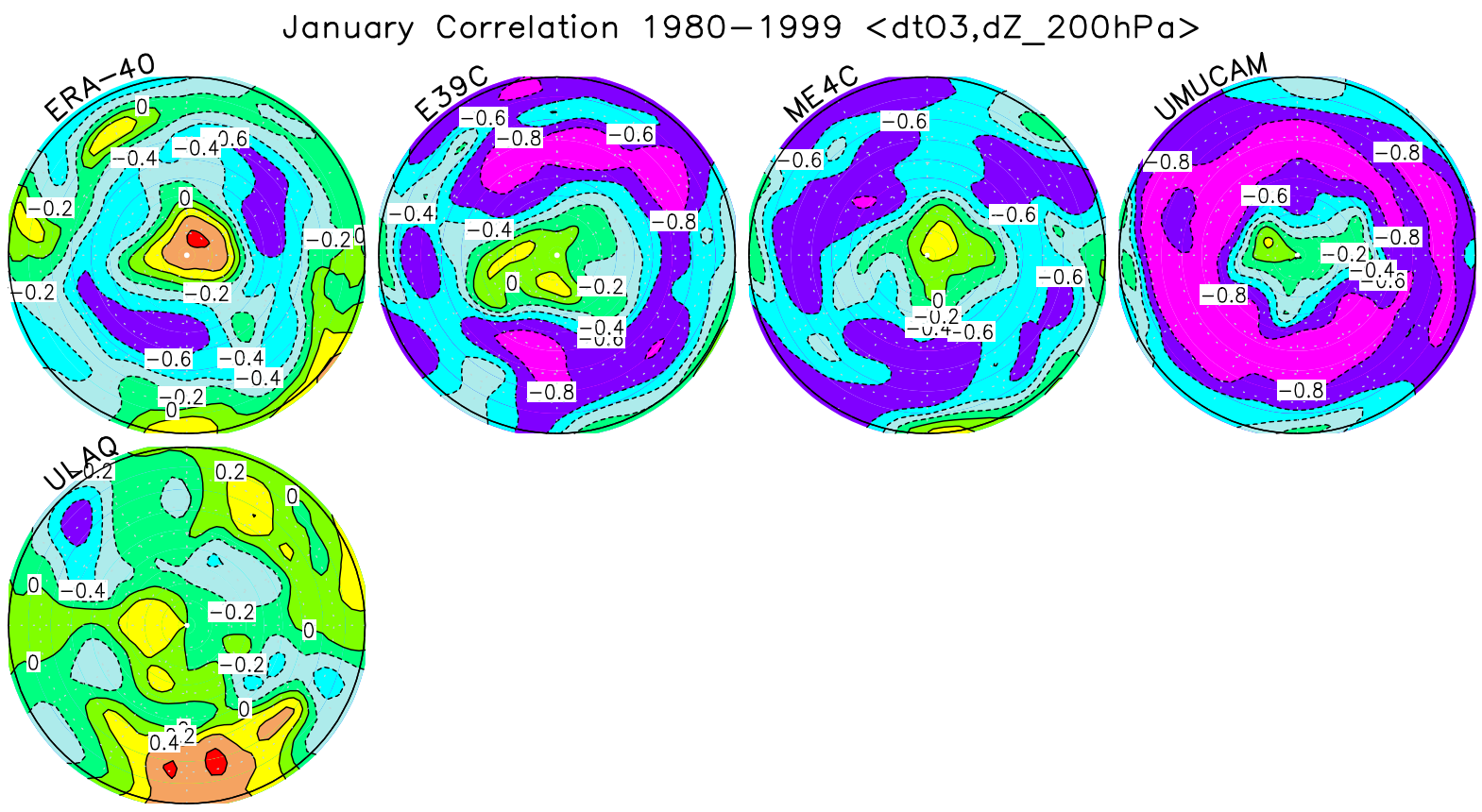

Fig. 2. Correlation between January monthly mean geopotential height anomalies at $200 \mathrm{hPa}$ and monthly mean total column ozone anomalies during the time period 1980-1999. Absolute values larger 0.44 can be considered to be significant at the $95 \%$ confidence level.

at $30 \mathrm{hPa}$ will relate to stronger/weaker vortices which are linked to lower/higher column ozone and thus a positive correlation should occur (e.g. Braesicke and Pyle, 2003). This is a combined effect of a suppressed/enhanced meridional circulation and a larger/smaller potential of chemical destruction due to lower/higher temperatures. A local high latitude impact from dynamics on column ozone is mediated by potential vorticity anomalies (e.g. Ambaum et al. , 2001 and Orsolini and Doblas-Reyes, 2003). For example a positive potential vorticity anomaly (in conjunction with a strong stratospheric vortex) is conjoined with upward bulging isentropes, a higher tropopause and lower column ozone. This effect counteracts the previous effect and positive correlations should be weak and small in spatial extent. To test for the link between column ozone and geopotential height anomalies we will calculate simple correlation maps first.

To advance our analysis, we have to establish the existence of known and well described leading modes of variability in the model systems analysed. Using northern hemisphere January monthly mean anomalies of geopotential height at 200 and $30 \mathrm{hPa}$ and column ozone we derive the leading EOFs and their temporal evolution. EOF1 for geopotential height anomalies is also known as the annular mode and is a well described structure in observations and in some model systems (Baldwin, 2001; Thompson and Wallace, 2001). Near the surface the annular mode shows some distinct asymmetries relating it to some classical meteorological indices like e.g. the North Atlantic Oscillation (NAO) (Wallace, 2000; Kodera and Kuroda, 2003). Higher up the name "annular mode" becomes more obvious because of the "very annular" nature of this mode of variability in the stratosphere. EOF2 in geopotential height anomalies in the free troposphere should reveal a tripole structure over the Pacific-North American (PNA) sector, which relates to the so-called PNA pattern (e.g. Wallace and Thompson, 2002) and a wave one structure (one maximum and one minimum in geopotential height anomalies along a longitude line) in the stratosphere. The existence of those spatial structures in the models is a prerequesit for successfully modelling the link between column ozone and geopotential height anomalies.

There is an ongoing debate about the physical nature of the statistically derived spatial patterns (EOFs) in the free troposphere. Christiansen (2002) argues for their physical nature, based on rotated EOFs at $500 \mathrm{hPa}$ and the fact that positive zonal mean wind anomalies in the stratosphere result in a larger probability for a positive annular mode phase at the surface 30 days later. This is in contrast to Ambaum et al. (2001) where the surface annular mode is described as a product of a mathematical method, and the NAO and PNA pattern are highlighted as the more physical relevant concepts. Even though this situation complicates the understanding of the physical causes of the differences in characteristic spatial patterns, it does not invalidate the attempt to use the patterns in comparing models and to judge them as similar or different.

Subsequently pointwise covariance maps of anomalies associated with EOFs 1 and 2 are calculated; between geopotential height anomalies at 200 and $30 \mathrm{hPa}$ and between 
Table 1. Summary of models in this comparison.

\begin{tabular}{llllll}
\hline Model & Horizontal res. $^{*}$ & No of levels & \multicolumn{4}{l}{ Uppermost mid-layer pressure } & Ozone chemistry \\
\hline ERA-40 & T159 & 60 & $0.1 \mathrm{hPa}$ & $(\sim 64 \mathrm{~km})$ & parameterised \\
E39C $(\mathrm{DLR})^{2}$ & T30 & 39 & $10.0 \mathrm{hPa}$ & $(\sim 32 \mathrm{~km})$ & comprehensive \\
ME4C $(\mathrm{MPI}-\mathrm{M} / \mathrm{C})^{3}$ & T30 & 39 & $0.01 \mathrm{hPa}$ & $(\sim 81 \mathrm{~km})$ & comprehensive \\
UMUCAM $^{4}$ & N48 & 58 & $0.1 \mathrm{hPa}$ & $(\sim 64 \mathrm{~km})$ & parameterised \\
ULAQ $^{5}$ & R6 & 26 & $0.04 \mathrm{hPa}$ & $(\sim 71 \mathrm{~km})$ & comprehensive \\
\hline
\end{tabular}

* The original spectral (T/R) or regular $(\mathrm{N})$ grid resolution is cited. The analysis grid is N48, see text.

${ }^{1}$ European Centre for Medium-Range Weather Forecasts (ECMWF) Re-Analysis

2 Deutsches Zentrum für Luft- und Raumfahrt-Institut für Physik der Atmosphäre

3 Max-Planck-Institut (MPI) für Meterologie and MPI für Chemie

${ }^{4}$ Unified Model University of Cambridge

5 Università degli Studi dell'Aquila

geopotential height anomalies at $200 \mathrm{hPa}$ and column ozone anomalies. In conjunction with the corresponding anomaly correlations we will be able to assess the relative strength of the mechanisms discussed above. There are two indicators we will compare:

- The spatial patterns of the scaled hemispheric covariance maps. How similar are the patterns between models and re-analysis data?

- The amplitude (absolute hemispheric maximum minus minimum) of the covariance patterns derived. How strong is the maximum local coherence/covariance between two levels/quantities?

This will help us to understand which leading modes of variability might be linked, either in terms of height or in terms of different quantities and how the relative importance of leading modes of variability differs in different model systems.

Section 2 details the models and data-sets used in this study and Sect. 3 will provide some more details about the chosen methodology and how it compares to other studies. After establishing the relation described above (Sect. 4) a comparison of characteristic spatial patterns (as approximated by the EOFs 1 and 2) for geopotential height anomalies at 200 and $30 \mathrm{hPa}$ and column ozone anomalies is presented in Sect. 5. The covariances between reconstructed anomalies between different levels or quantities are discussed in Sect. 6. Section 7 will provide a summary and conclusions.

\section{Models and data}

For the period considered, 1980-1999, we compare four different CCMs and the largely consistent assimilated ERA40 data-set (Uppala et al., 2005). To some extent we have to consider ERA-40 as a "proxy of observations" because it assimilates meteorology and ozone during the time period of interest, but there are particularly some limitations to the assimilation of ozone (Dethof and Holm, 2004). The main ozone constraint is derived from TOMS column measurements, therefore a lot of a-priori profile information is maintained and during polar night ozone in high latitudes is not constraint by observations due to a lack of measurements. Nevertheless, by the very nature of the assimilation scheme used, column ozone (where measured) is nearly identical to TOMS. Problems may arise in high latitudes on the winter hemisphere, when the model relies on the parameterised ozone chemistry alone (a Cariolle scheme, Cariolle and Déqué, 1986) in conjunction with a simple temperature dependent parameterisation representing additional ozone loss due to chlorine activation on polar stratospheric clouds). Due to this uncertainty it is not possible to interpret ECMWF fully as an observational data set, but it can be used as a largely well constraint climate model.

The CCM data-sets used in this study are the result of model integrations attempting to represent the time period from 1980-1999 (note that we use a subset of models featured in Eyring et al., 2006). Table 1 presents a brief model summary. As can be seen from the table the range of models is quite diverse (in this context we refer to ERA-40 as a model as well, even though it will be used as an observationsal proxy). To make the intercomparison easier we use a common diagnostic grid for all calculations (note that tests using the original model grids showed no depence of the results on the grid). All model data is interpolated to the N48 grid used by the UMUCAM model, which corresponds to a resolution of $3.75^{\circ}$ in longitude by $2.5^{\circ}$ in latitude on the required pressure levels.

All CCMs we are assessing here treat ozone in the stratosphere as an interactive trace gas. Some other gases (like CFCs) might be prescribed. The models have either performed fully transient runs (E39C, ME4C, ULAQ) or they include a transient component and fix certain other parameters to typical 1990s values (UMUCAM). The E39C, ME4C and ULAQ runs have been designed to be as realistic as possible in their representation of the 1980-1999 time period 


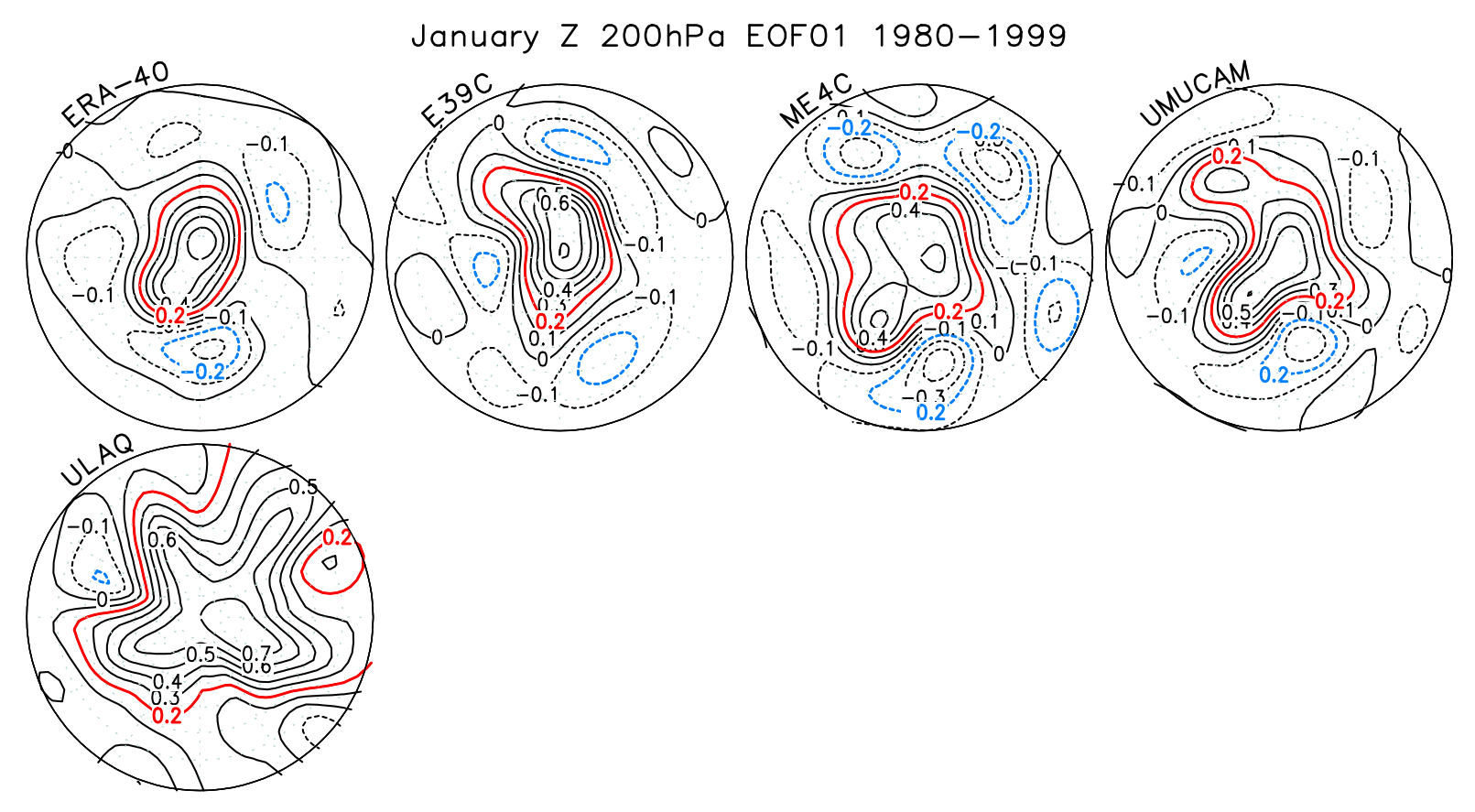

Fig. 3. EOF1 in geopotential height at $200 \mathrm{hPa}$ for January.

using a multitude of specified time varying external forcings. The UMUCAM run was deliberately not designed as a typical scenario integration and uses time varying sea surface temperatures only (other external forcings are set to typical 1990s values) to allow for the easier assessment of selected sensitivities. Note that all models prescribe observed monthly mean sea surface temperatures and calculate surface pressure, except the ULAQ model, where the surface pressure is fixed to $1000 \mathrm{hPa}$. This difference is linked to the form of model equations solved, with all models being based on the full set of primitive equations, except the ULAQ model which uses a quasi-geostrophic form of the primitive equations. Details of the models are given in the following papers: E39C (DLR): Dameris et al. (2005, 2006); ME4C (MPI-M/C): Manzini et al. (2003) and Steil et al. (2003); UMUCAM: Braesicke and Pyle (2003, 2004); ULAQ: Pitari et al. (2002). It is interesting to note that most models here are spectral models, solving the equations of motion in wavenumber space. Only UMUCAM is a gridpoint model and does not employ transformations between wavenumber and gridpoint space. In addition, it should be noted that E39C and ME4C are based on the same original model and have mainly deviated by the employed transport scheme and developments of the vertical domain modelled. Here, we assess the interannual variability under the assumption that details of the boundary forcings are not important and that changes in time varying boundary forcings will more stronlgy affect trends. We will return to this assumption later in the conclusions.
Table 2. Pattern correlations for geopotential height EOF1 and EOF2 at $200 \mathrm{hPa}$. The upper triangle (light gray shading) is for EOF1, the lower triangle (unshaded) is for EOF2. The exact threshold for statistical significance is hard to establish, because the correct number of degrees of freedom cannot be established easily. Therefore a subjective highlighting (values above $\geq 0.5$ are in bold) is used as a crude measure of similarity.

\begin{tabular}{lccclcl}
\hline Model & ERA-40 & E39C & ME4C & UMUCAM & ULAQ & EOF1 \\
\hline ERA-40 & 1.0 & $\mathbf{0 . 8 5}$ & $\mathbf{0 . 8 3}$ & $\mathbf{0 . 8 9}$ & 0.40 & ERA-40 \\
E39C & 0.26 & 1.0 & $\mathbf{0 . 8 1}$ & $\mathbf{0 . 8 3}$ & $\mathbf{0 . 5 5}$ & E39C \\
ME4C & 0.26 & 0.19 & 1.0 & $\mathbf{0 . 8 5}$ & 0.49 & ME4C \\
UMUCAM & 0.41 & $\mathbf{0 . 6 6}$ & 0.06 & 1.0 & $\mathbf{0 . 6 5}$ & UMUCAM \\
ULAQ & -0.12 & -0.24 & -0.19 & -0.14 & 1.0 & ULAQ \\
\hline EOF2 & ERA-40 & E39C & ME4C & UMUCAM & ULAQ & Model \\
\hline
\end{tabular}

\section{Methodology}

One simple mechanism for varying column ozone is the change of tropopause heights in middle latitudes (e.g. Dobson, 1930; Orsolini et al., 1998 and Steinbrecht et al., 1998). The change in tropopause heights is also mirrored in geopotential height anomalies at a pressure surface close to the tropopause (e.g. $200 \mathrm{hPa}$ ). Even though this effect is most pronounced in middle latitudes, a correlation between column ozone and geopotential height anomalies can be derived anywhere. This concept of "vertical coherence" (e.g. high tropopause/low column ozone or vice versa) can also be extended towards geopotential height anomalies at different pressure levels. Note that this differs from other approaches 
looking into interrelations between geopotential heights on pressure levels as e.g. used by Perlwitz et al. (2000).

Note that the methodology does not enable us to find a physical rationale for the characteristic spatial patterns derived (e.g. Wallace, 2000; Ambaum et al., 2001; Wallace and Thompson, 2002). We are focusing on the comparison of results between a data assimilation system (as our best guess of observed interannual variability between 1980-1999, with the above mentioned limitations in ozone) and models trying to capture the characteristics of interannual variability between 1980 and 1999. Unlike Steinbrecht et al. (2006) we do not attempt the attribution of interannual variability to forcing parameters in a regression model, but we try to unravel the functioning of the coupled variability (between different height regimes) in the models. We assume that similiarities in interannual variability will manifest themselves in similar patterns and that deviations from the patterns are linked to deficits or differences in the model systems.

We use monthly mean anomalies of geopotential height and column ozone (in addition we diagnose partial column ozone between 380 and $550 \mathrm{~K}$ isentropic temperature levels) and evaluate the relationship between geopotential height and column ozone anomalies by statistical means. As already mentioned in the introduction, we will go through a three step process to assess the links between column ozone and meteorology: First, we will use point-by-point correlations between monthly mean anomalies of geopoptential heights at selected pressure levels and ozone columns to discuss the idea of vertical coherence as explained above. To establish the overall relation of different anomaly time-series, correlation coefficients are more intuitive. For the reconstructed anomalies discussed later the standard deviations can become regionally very small due to the fixed position of zero lines (given by the characterisitc spatial patterns, EOFs) and therefore correlation coefficients are no longer well defined. Correlations and covariances are related through a scaling with the product of the standard deviations and therefore covariances are shown. This deliberately simple approach is not limiting the ability to discuss pattern similarities between models, or to pinpoint regions where the concept of local coherence holds very well or not at all for a given large scale feature. We will make this clear by contrasting our approach with results from literature. Secondly, a detailed investigation of characteristic spatial patterns for the anomaly fields will use the two leading EOFs of geopotential height anomalies at different pressure levels and column ozone. We use all anomalies available on the northern hemisphere, unweighted but interpolated to a common horizontal grid (see above). A sensitivity check applying latitudinal weighting left our conclusions unchanged. Thirdly, a detailed discussion of the point-by-point covariance patterns of reconstructed anomalies in geopotential heights and ozone columns using the two leading modes of interannual variability (EOFs 1 and 2) follows. Note that we focus solely on the interannual variability. No assessment of trends (which are removed prior to the fur- ther analysis) or shifts in climate regimes will be conducted. We assume that the first two EOFs are the same over the time period evaluated (20 years) and assess whether the relation between interannual changes in meteorology and column ozone is reproduced in a similar way in the CCMs and the re-analysis data.

\section{Anomaly correlations}

To illustrate the general behaviour of the models in terms of vertical coherence and their relationship between column ozone and meteorology (as represented by the interannual variation of $200 \mathrm{hPa}$ geopotential height) we will discuss linear correlations between monthly mean anomalies. The correlation maps are only used to give us some indication of overall behaviour; they are certainly no measure of cause and effect, but with an underlying idea of how meteorology is linking different levels of the atmosphere and how column ozone is affected by changes in e.g. tropopause height or vortex strength (see introduction), we will be able to interpret and compare the resulting patterns.

Figure 1 shows the correlation between January monthly mean geopotential height anomalies at 200 and $30 \mathrm{hPa}$ during the time period 1980-1999. We know that the interannual variability at $30 \mathrm{hPa}$ relates to the characteristics of the winter vortex and there is an amount of coherence between the mid-winter vortex in the stratosphere and the geopotential height anomalies in the upper troposphere. We find reasonable agreement between the model data (E39C, ME4C and UMUCAM) and the analysis (ERA-40). All show high positive correlations in high latitudes but the annularity and the absolute amplitude of the patterns are different, with the analysis showing the highest correlations. The ULAQ model shows a very weak signal only in high latitudes with only a small area of positive correlation.

Figure 2 shows the correlation between January monthly mean geopotential height anomalies at $200 \mathrm{hPa}$ and monthly mean column ozone anomalies during the time period 1980 1999. There are two distinct regimes visible in the correlations: a middle latitude one with negative correlation and a polar one with positive correlation. The patterns are more pronounced in the CCMs solving the primitive equations with prescribed boundary forcings than in the analysis or in the ULAQ model (see above). Nevertheless the overall agreement between ERA-40 and E39C, ME4C and UMUCAM is good.

As mentioned in the introduction, the reason for these two regimes can be understood physically: In middle latitudes column ozone variability on many timescales is to some extent controlled by the tropopause height which is correlated to the height anomaly at $200 \mathrm{hPa}$. A positive height anomaly (a higher than average tropopause) is related to lower than average column ozone and vice versa leading to a negative correlation. In high latitudes meridional transport and the 


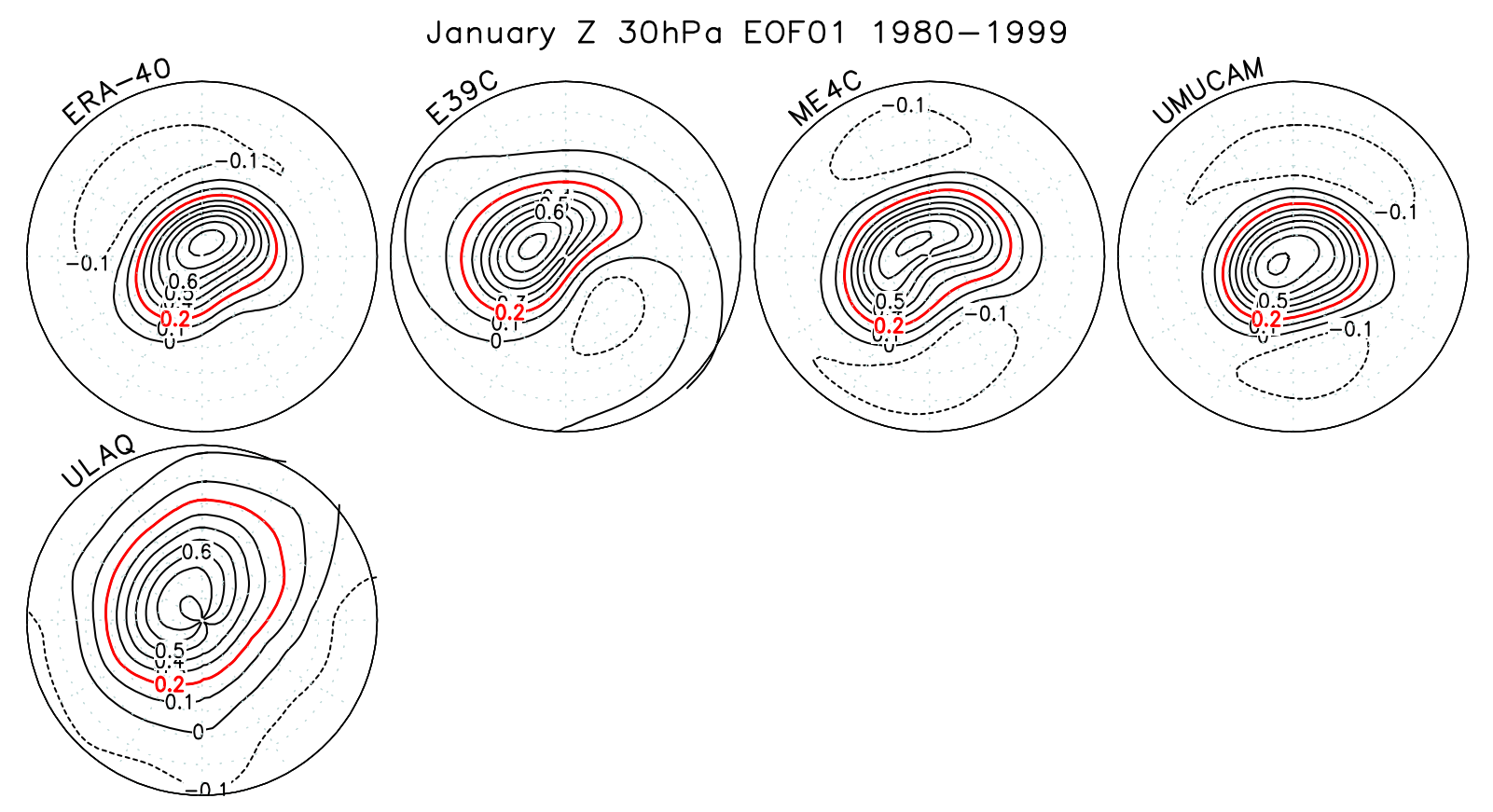

Fig. 4. EOF1 in geopotential height at $30 \mathrm{hPa}$ for January.

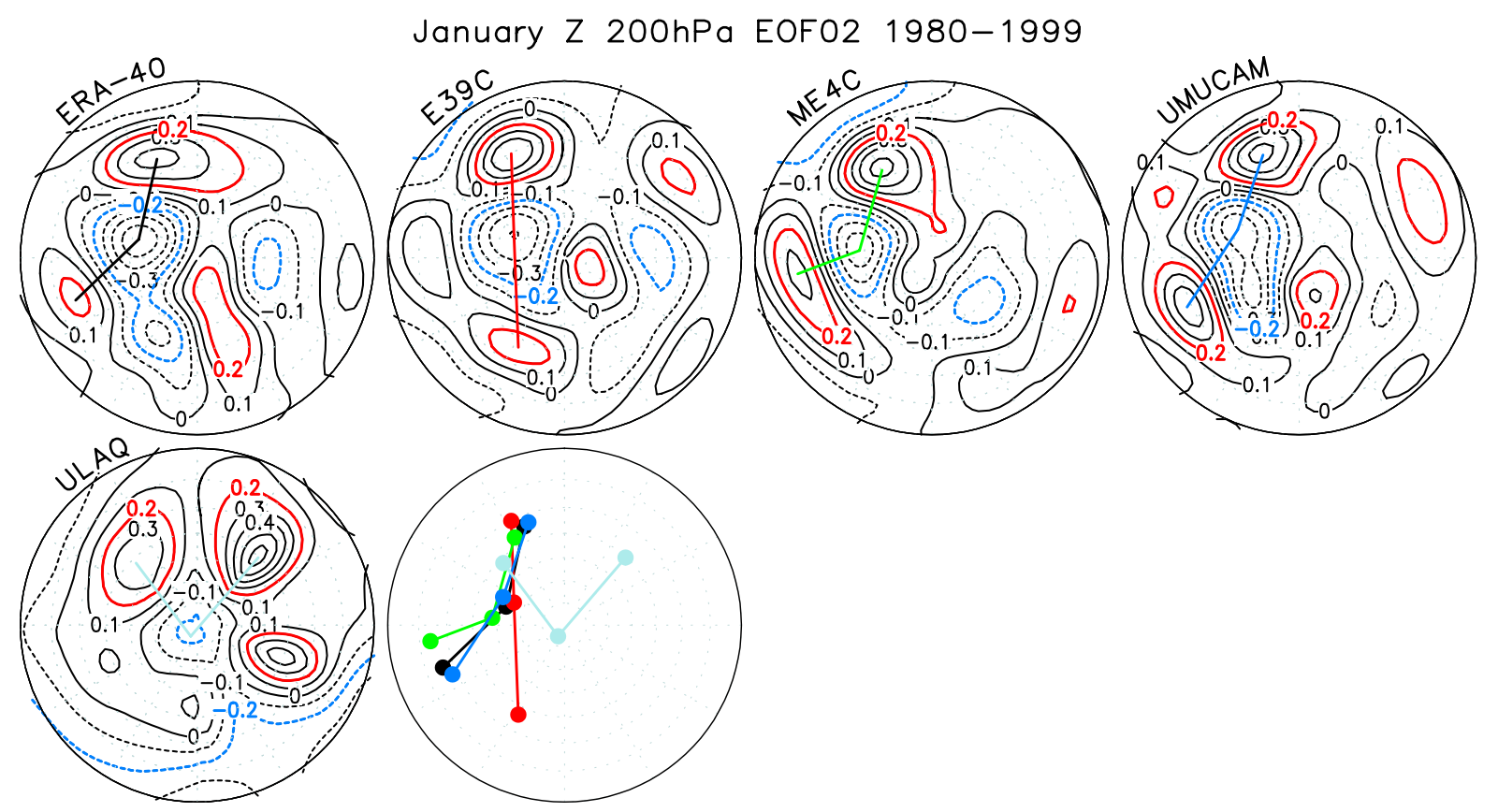

Fig. 5. EOF2 in geopotential height at $200 \mathrm{hPa}$ for January and position markers for the PNA tripole (repeated in the lower right plot). 
strength of the polar vortex are more important in controlling the column ozone abundance. A stronger than average vortex (linked to a negative polar height anomaly, see above) is likely to suppress meridional transport and leads therefore to lower polar column ozone and vice versa. This control mechanism is then indicated by a positive correlation pattern in high latitudes (see Sect. 1 for further details).

Using the partial ozone column between 380 and $550 \mathrm{~K}$ (most ozone contributing to the total column will be located in this region) instead of the total ozone column does not change the overall behaviour as discussed in conjunction with Fig. 2. A small amount of noise becomes apparent due to the fact that the partial ozone column is derived from pressure gridded ozone mixing ratios.

In this section we have confirmed a general picture of the vertical coherence of the models during January on the northern hemisphere and a conceptual interpretation of the simple link between column ozone and meteorology (as represented by $200 \mathrm{hPa}$ geopotential height anomalies). In the following we attempt to split this general overall behaviour into components related to the leading modes of variability in each model system using an EOF analysis.

\section{Leading EOFs of heights and column ozone}

\subsection{EOFs in geopotential heights}

As motivated in section 1 we focus on January and discuss the spatial patterns of the EOFs for geopotential height and column ozone anomalies. Thereafter we discuss the spatial patterns and amplitudes of covariances calculated using reconstructed anomalies of geopotential height and column ozone for individual leading modes of variability (the focus will be on EOFs 1 and 2 and their associated time evolution and weights).

\subsubsection{The vertical structure of the annular mode}

We find in the lower free troposphere an annular structure centred over the pole with a marked asymmetry over the Atlantic-West European sector in all models (not shown). The asymmetry is related to the NAO. Schnadt and Dameris (2003) discuss the relationship between the NAO and column ozone recovery in E39C and find a decrease of the NAO index in a future climate in conjunction with a stronger dynamical heating in the stratosphere. In addition, Braesicke et al. (2003) analyses the NAO signature in column ozone for two different models, including UMUCAM. There is another asymmetry in most models (including ERA-40) towards the Pacific sector. This asymmetry is most pronounced in ME4C. The asymmetries are generally weak in the ULAQ model, presumably related to the fixed surface pressure and the lower horizontal resolution.

Figure 3 shows EOF1 in geopotential height at $200 \mathrm{hPa}$ for January. The polar annular structure is already smoother compared to further down but pronounced asymmetries can be seen. The one identified in the Atlantic-West European sector is still apparent and there is a pronounced anomaly in the Pacific-Asian sector. The CCMs with a resolution above or equal to T30 compare well with the ERA-40 anomalies.

Figure 4 shows EOF1 in geopotential height at $30 \mathrm{hPa}$ for January. The two models with a higher upper boundary and higher horizontal resolution (ME4C and UMUCAM) show two distinct minima in middle latitudes, whereas only one minimum is seen in ERA-40. In general this plot reveals the climatological position of the polar vortex during Januray in the models. Note that all troposphere-resolving CCMs show a clear shift of the polar vortex towards the Atlantic/West European sector, but E39C shows a displacement of the annular mode pattern towards the North American sector.

The models with variable surface pressure (E39C, ME4C and UMUCAM) show a good comparison with observations (ERA-40). The model with a fixed surface pressure (ULAQ) has some problems with the tropospheric annular mode and the NAO related asymmetries, but does perform well in the stratosphere.

\subsubsection{EOF2 at selected pressure heights}

Figure 5 shows EOF2 in geopotential height at $200 \mathrm{hPa}$ for January. Much more small scale stucture is obvious as compared to EOF1. A prominent feature is a tripole over the Pacific-North American sector, which relates to the so-called PNA pattern (e.g. Wallace and Thompson, 2002). Wallace and Thompson (2002) discussed this pattern in their Fig. 4, derived by regressing the second principal component (PC2) of surface level pressure anomalies onto geopotential height anomalies at $500 \mathrm{hPa}$. Because we focus on geopotential height changes and their local impact on column ozone amounts, we focus on lower pressures (larger altitudes) compared to Christiansen (2002) (see Sect. 1) to better approximate local tropopause changes. In addition our data base is sparser (monthly mean data compared to daily 30-day low pass filtered) and we therefore focus clearly on the interannual timescale and not on smoothly and continuously varying data. Given the nature of our data and the chosen pressure surface we do not require a rotation of the EOFs to reveal the PNA pattern. To highlight the relative position of the PNA patterns in each model the strongest maxima relating to the tripole structure are marked out with connecting lines, which are repeated on a common map in Fig. 5. The agreement between ME4C and UMUCAM is quite striking, given that they are very different models in terms of their model formulation (spectral versus gridpoint, different choice of prognostic variables, etc.). E39C displays a slightly more elongated tripole structure reaching more into the Atlantic sector (see comparison of positions of extrema in the lower right part of Fig. 5). In addition to the tripole/PNA structure ERA-40 also indicates a second tripole structur in the Atlantic-European sector which cannot be so readily identified in E39C, ME4C 


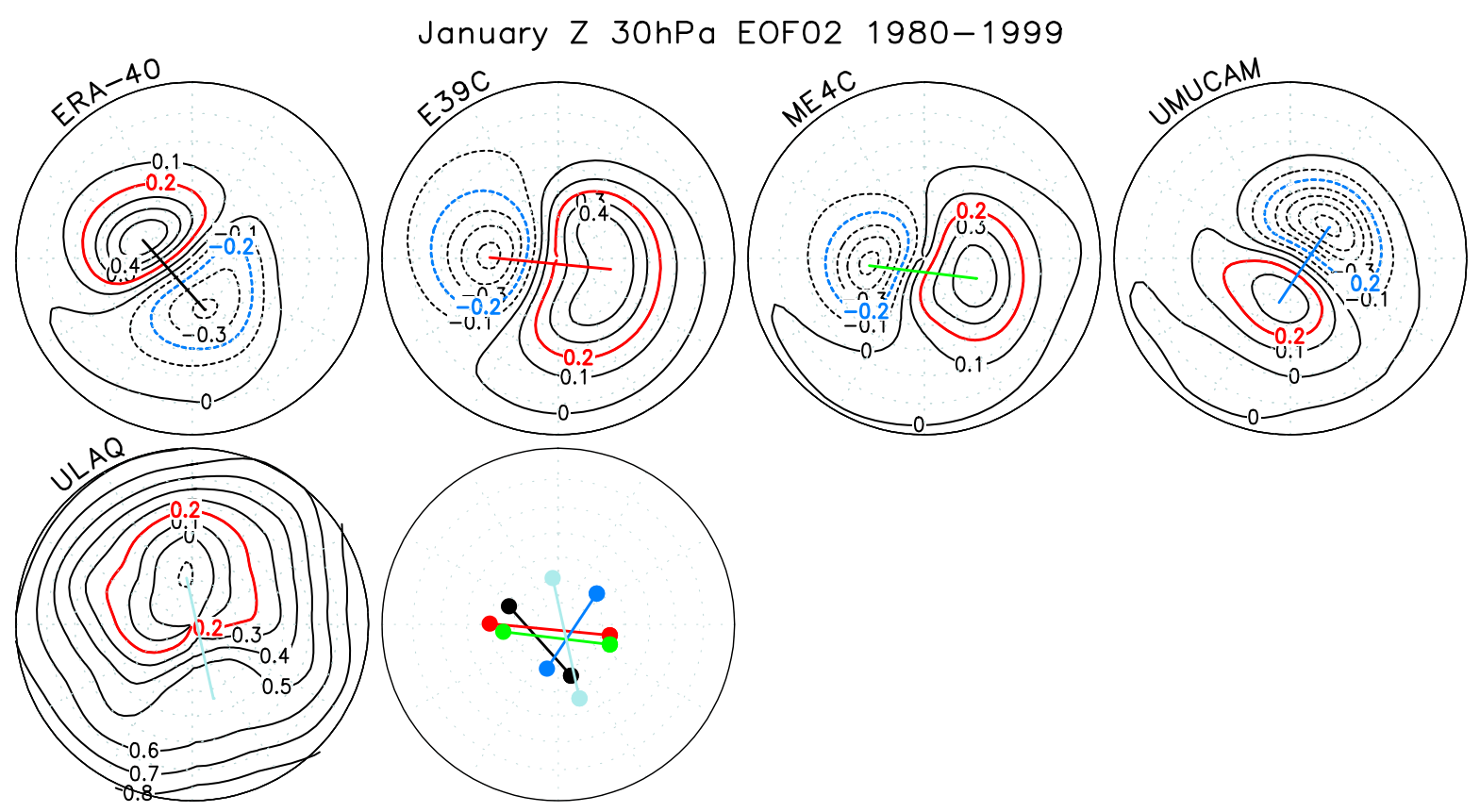

Fig. 6. EOF2 in geopotential height at $30 \mathrm{hPa}$ for January and position markers for the minimum and maximum of EOF2 (repeated in the lower right plot).

and the UMUCAM model. The ULAQ model also shows smaller scale features in EOF2, but the position of the features do not relate well to the observations or other CCMs.

As illustrated in Table 2, area weighted spatial correlations for a simple (more annular) spatial structure like EOF1 are generally high between the models. In contrast, the correlations for EOF2 are generally much smaller, with UMUCAM and $\mathrm{E} 39 \mathrm{C}$ being the most similar. Even though the agreement in the North American/Pacific sector is very good between ME4C and UMUCAM, the correlation is brought down by the out-of-phase (but low amplitude) behaviour in other sectors. Note also that the wavetrain for the ULAQ model is very different from ERA-40 and correlations are therefore weakly negative.

Figure 6 shows EOF2 in geopotential height at $30 \mathrm{hPa}$ for January. All models show a strong "wavenumber 1" structure (one minimum and one maximum along a longitude line), apart from the ULAQ model where the "wavenumber 1 " structure is only unincisive. The phase of the anomalies (position of the absolute minimum and maximum, see the lower right plot in Fig. 6) differ substantially between all CCMs and ERA-40, with E39C and ME4C displaying some agreement.

\subsection{EOFs in total and partial column ozone}

Figure 7 shows EOF1 in column ozone for January. Note, that even though the ERA-40 column ozone data in lower latitudes is constrained by the assimilation of column ozone observed from the TOMS instrument that constraint is not avail- able during polar night in high latitudes where the TOMS instrument cannot measure due to the unavailability of light (see detail above about the parameterised ozone scheme used). EOF1 in column ozone as provided by the ERA-40 data shows a very wide annular mode with a strongly confined outer gradient region. This feature might be partially due to the assimilation system, switching over from an area with TOMS data to an area without TOMS data assimilation. All models do have an annular mode structure in column ozone as well, but slightly more confined towards polar latitudes. E39C and ME4C do show a more elongated pattern than the UMUCAM and ULAQ models.

EOF1 in partial column ozone $(380-550 \mathrm{~K})$ for January (not shown) compares well to Fig. 7 showing column ozone. The ERA-40 pattern appears to widen and an elongated core region appears. Interestingly, in E39C and ME4C the annular pattern shrinks and the elongation of the dominant pattern is more apparent, whereas the UMUCAM and ULAQ models are still fairly annular. Certainly those features depend crucially on the modelled ozone profiles and their relative positions with respect to the isentropic levels chosen. There is some kind of family similarity between the E39C and ME4C models, both using the same dynamical core and similar chemistry, implying that the result depends more on the troposphere and is not influenced by the different choice of upper boundaries. Interestingly, the UMUCAM model with complex dynamics but simple chemistry and the ULAQ model with simple dynamics and complex chemistry show a similar more annular pattern compared to E39C and ME4C. The ERA-40 result is difficult to interpret; the pattern widens 

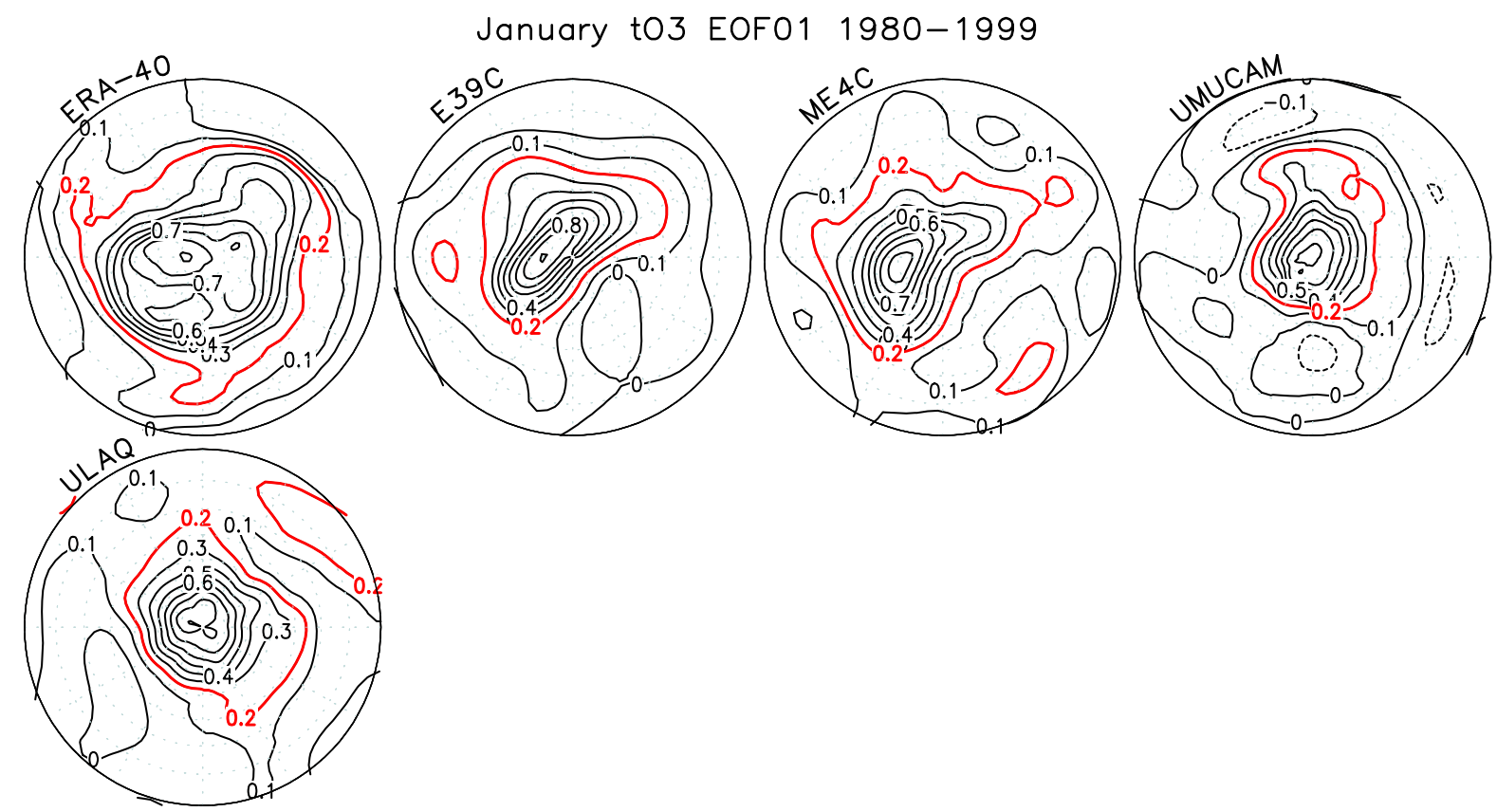

Fig. 7. EOF1 in total ozone for January.
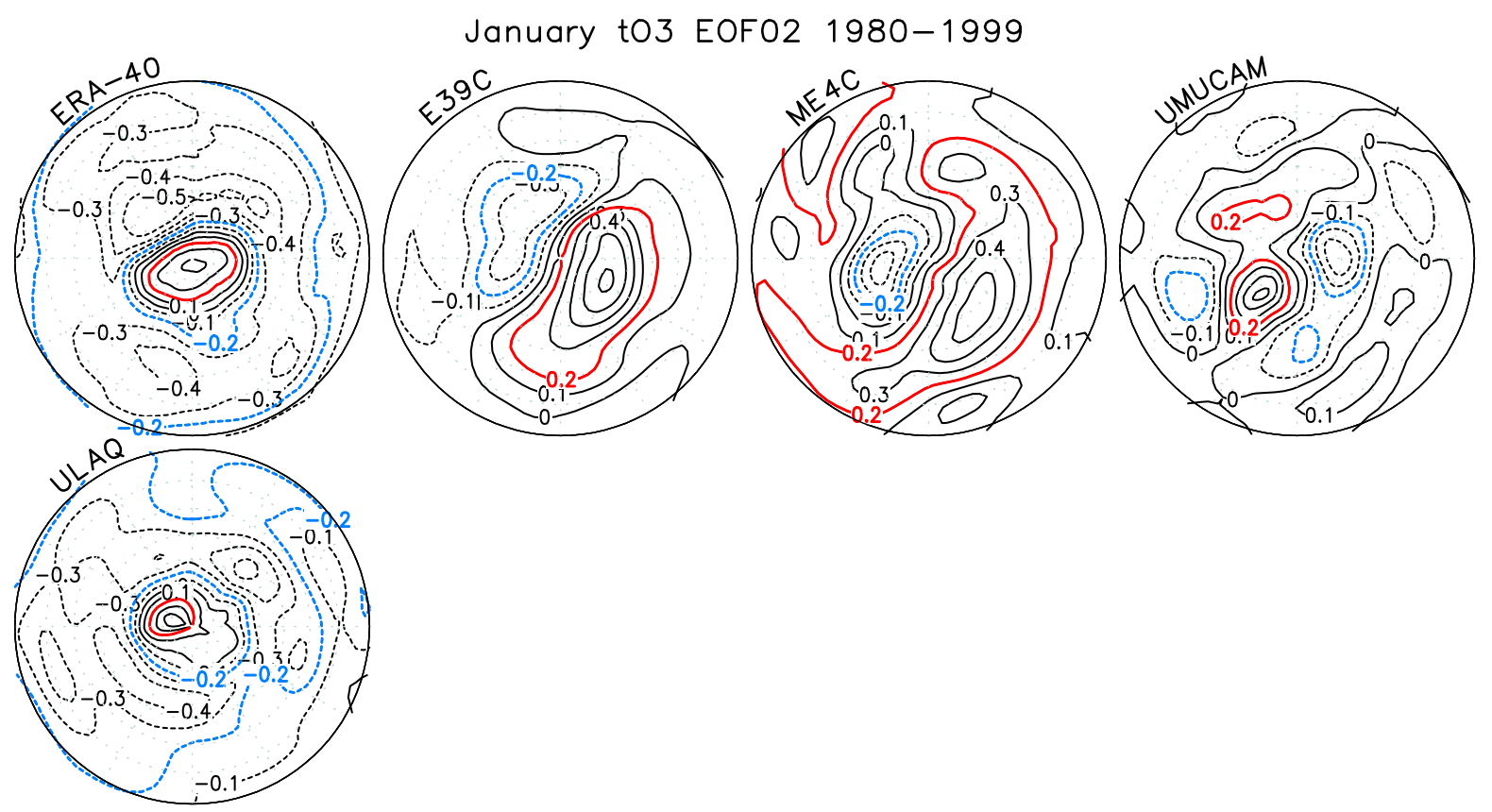

Fig. 8. EOF2 in total ozone for January. 


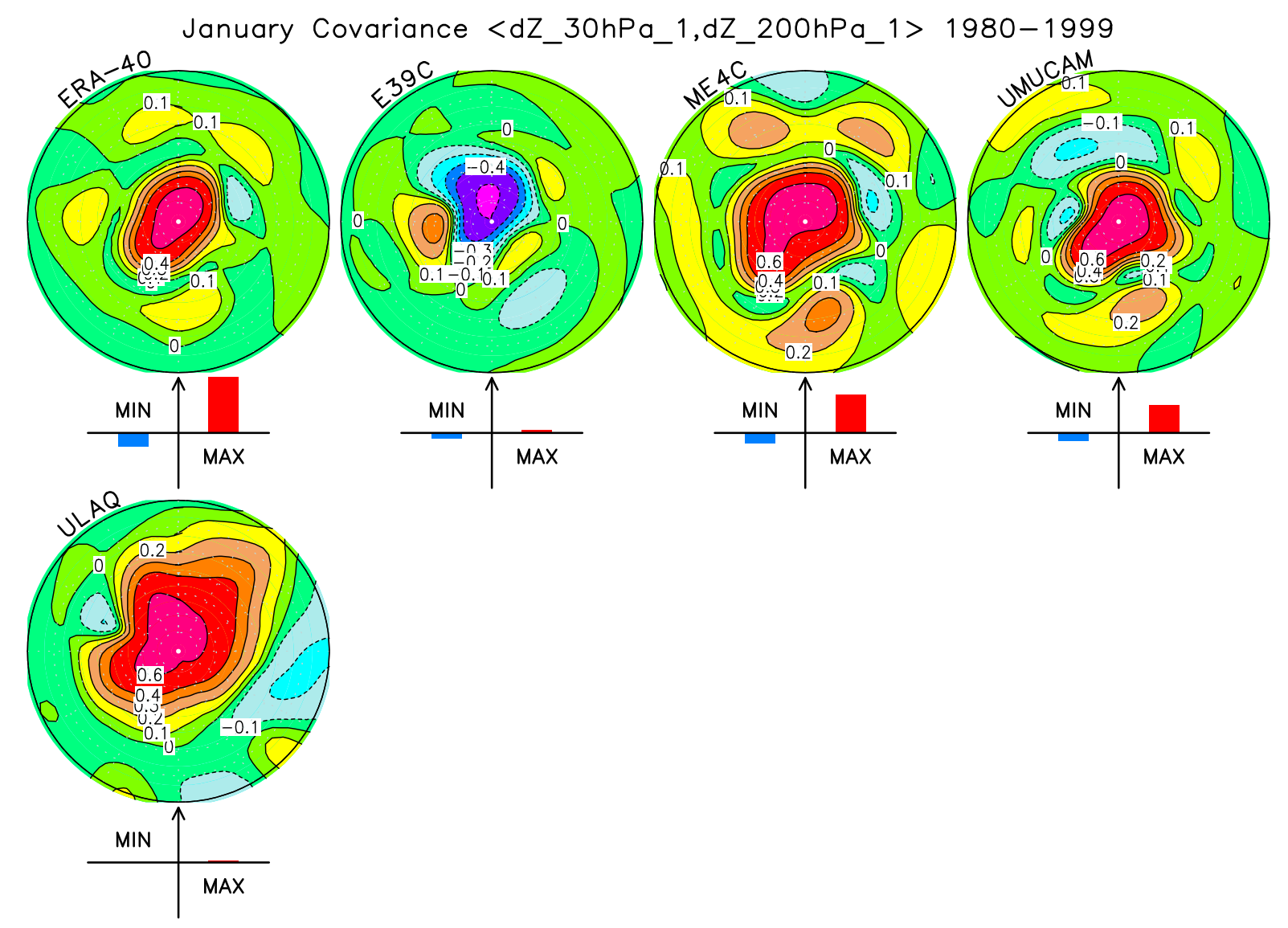

Fig. 9. Covariance of reconstructed geopotential height anomalies at 30 (EOF1) and $200 \mathrm{hPa}$ (EOF1) for January.

and even though it does show some elongation the pattern is less well defined and shows a different orientation to E39C and $\mathrm{ME} 4 \mathrm{C}$ results. In addition, the pattern reaches far out into low latitudes, which is not seen in any of the models. As mentioned earlier, this behaviour may be caused in part by the assumptions made in the data assimilation scheme on how to distribute the measured TOMS column ozone data vertically. Note that these differences have not affected the correlations between geopotential height and (partial) column ozone anomalies as discussed in Sect. 4.

Figure 8 shows EOF2 in column ozone for January. ERA40 and the ULAQ model seem to show some compensation pattern with respect to EOF1 which is still fairly annular, whereas E39C and ME4C show a well defined dipole structure with a very similar orientation. The UMUCAM model indicates a tripole structure leading from North America over the Pacific towards Russia.

EOF2 in partial column ozone for January (not shown) reveals a largely similar behaviour compared to the column ozone. E39C still shows a clear dipole structure whereas ME4C now indicates a tripole structure reaching from the American sector towards the Atlantic-West European sector. A very similar pattern is found in the UMUCAM model with a weaker second tripole adjacent to the dominant one.

For EOF1 in column ozone the four CCMs are similar. All show a fairly annular mode confined to polar latitudes. Interestingly, ERA-40 indicates a much wider annular mode. For the partial column ozone the ERA-40 structure widens even more, but the models are now clearly in two groups, either showing a confined elongated pattern (E39C, ME4C) or a more annular behaviour (UMUCAM, ULAQ). The behaviour for EOF2 is less conclusive and more varied.

\section{Covariances for reconstructed anomalies}

A simple measure of vertical coherence as explained in Sect. 3 is explored. The covariance between two reconstructed time series (as given by the product of EOF (spatial), principal component (PC, temporal) and weight) is calculated and presented as a map. In addition, we compare this approach to coupled mode analysis available in the literature. To provide the important information in a compact form we will only show maps for EOF1-EOF1 covariances; the other possible combinations are summarised in bar charts showing amplitudes only. 
$<d Z$ 30hPa_x,dZ_200hPa_y $>$

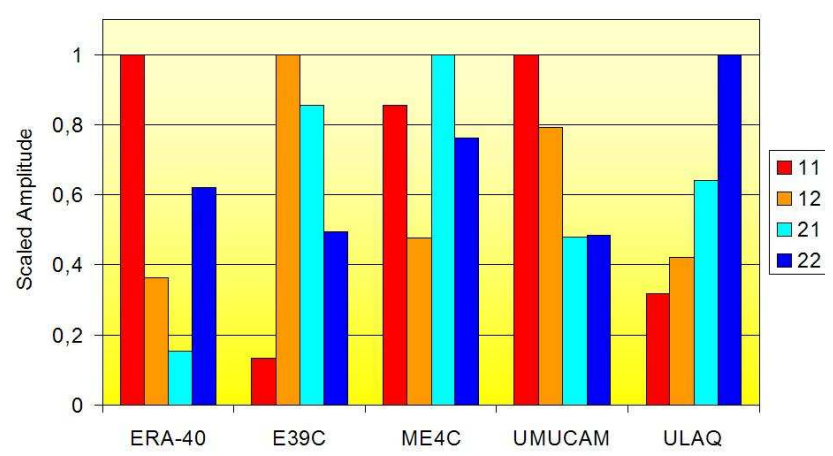

Fig. 10. Amplitudes of geopotential height covariance patterns scaled with the maximum amplitude found in each model.

\subsection{Covariances for height anomalies at different pressures}

Figure 9 shows the covariance of reconstructed geopotential height anomalies at 30 (EOF1) and $200 \mathrm{hPa}$ (EOF1) for January. As expected from the straightforward covariance approach discussed earlier we find a polar annular region of positive covariance in ERA-40 with a significant amplitude. This feature is also seen in ME4C and the UMUCAM model (both resolving the stratosphere), but with a slightly weaker amplitude. The ULAQ model indicates a larger area of positive covariance but with no significant amplitude, whereas E39C shows a small polar region of negative covariance surrounded by small areas of positive correlations but similar to the ULAQ model the amplitude is very low. All models including a comprehensive stratosphere show a positive coherence/covariance between the 30 and $200 \mathrm{hPa}$ levels (but with the ULAQ model not showing a significant amplitude). E39C with a low upper lid displays a pattern of opposite sign but shows also a very low amplitude, hinting towards a very weak coherence.

Figure 10 compares the relative amplitude distribution for the covariance patterns in each model system. Note that the bars are now scaled against the maximum amplitude found in each individual model. The numbers in the legend to the right refer to the $\mathrm{x}$ and $\mathrm{y}$ place holders in the bar graph title, identifying the pair of EOFs used to calculate the covariance amplitudes with respect to the earlier figures. ERA-40 shows the largest amplitudes for covariance patterns calculated with the same order (e.g. EOF1-EOF1 (11) or EOF2-EOF2 (22)) at the two different heights considered. This is in good agreement with Perlwitz and Graf (1995) and their description of two coupled natural modes during $\mathrm{NH}$ winter, one describing the link between stratospheric vortex strength and tropospheric circulation over the North Atlantic (11) (this link has been recently re-examined by Walter and Graf, 2005 and Graf and Walter, 2005) and the other linking the stratospheric zonal wavenumber 1 with a PNA-like pattern in the stratosphere (22). None of the models reproduce this clear sepa- ration in the amplitude distribution. E39C has strongest amplitudes for the mixed modes (12) and (21). This is less obvious in ME4C which shows a stronger (11) covariance amplitude. UMUCAM shows the strongest amplitude for (11) as in ERA-40, but drops of towards higher orders, whereas ULAQ shows the converse behaviour.

In general, most models display a reasonable amount of vertical coupling (e.g. a significant amplitude in the covariance), with the ULAQ model showing the weakest vertical coherence. E39C tends towards coupling involving higher tropospheric EOFs (EOF1-EOF2 coupling) to reproduce the overall positive correlation in polar latitudes between tropospheric and stratospheric polar height anomalies, whereas ME4C and the UMUCAM model both show a clear EOF1EOF1 coupling.

\subsection{Covariances for column ozone and height anomalies}

Here, we will evaluate the relationship between (partial) column ozone anomalies and geopotential height anomalies at $200 \mathrm{hPa}$.

Figure 11 shows the covariance of reconstructed geopotential height anomalies at $200 \mathrm{hPa}(\mathrm{EOF} 1)$ and partial column ozone anomalies (EOF1) for January. Even though the partial column ozone EOF1 derived from ERA-40 data is wide, a well defined annular region of positive covariance in polar latitudes surrounded by some smaller negative anomalies is apparent. The shape of the anomalies in the CCMs is largely determined by the column ozone EOF1 pattern. The covariances are fairly annular for UMUCAM and ULAQ and elongated for E39C and ME4C. The phase problem identified earlier in the geopotential height analysis is now apparent again in the E39C results. Note that all CCMs have a much smaller amplitude than ERA-40. The weak negative covariances in low latitudes seem to support the idea that the meridional motion in conjunction with the vortex strength (EOF1 for geopotential heights should be a good proxy of the overall vortex strength, see descussion of annular modes above) is regulating high latitude ozone on interannual timescales, but does not hugely affect lower latitudes where "tropospheric weather" (tropopause height as e.g. approximated by $200 \mathrm{hPa}$ geopotential height anomalies) is more important. This modulation of the poleward meridional transport might be less well represented in E39C due to the lower upper boundary. This is also in agreement with Braesicke and Pyle (2003), in which the best proxy for the UMUCAM vortex strength with respect to column ozone in high latitudes was identfied as the $60^{\circ} \mathrm{N}, 10 \mathrm{hPa}$ zonal-mean zonal wind, indicating that transport processes in and around this level are important to maintain the correlation.

Figure 12 compares the relative amplitude distribution for the covariance patterns in each model system. It is organised like Fig. 10, but shows the covariance amplitudes for partial ozone columns and geopotential heights at $200 \mathrm{hPa}$. ERA-40 shows the largest amplitude for the covariance pattern calcu- 


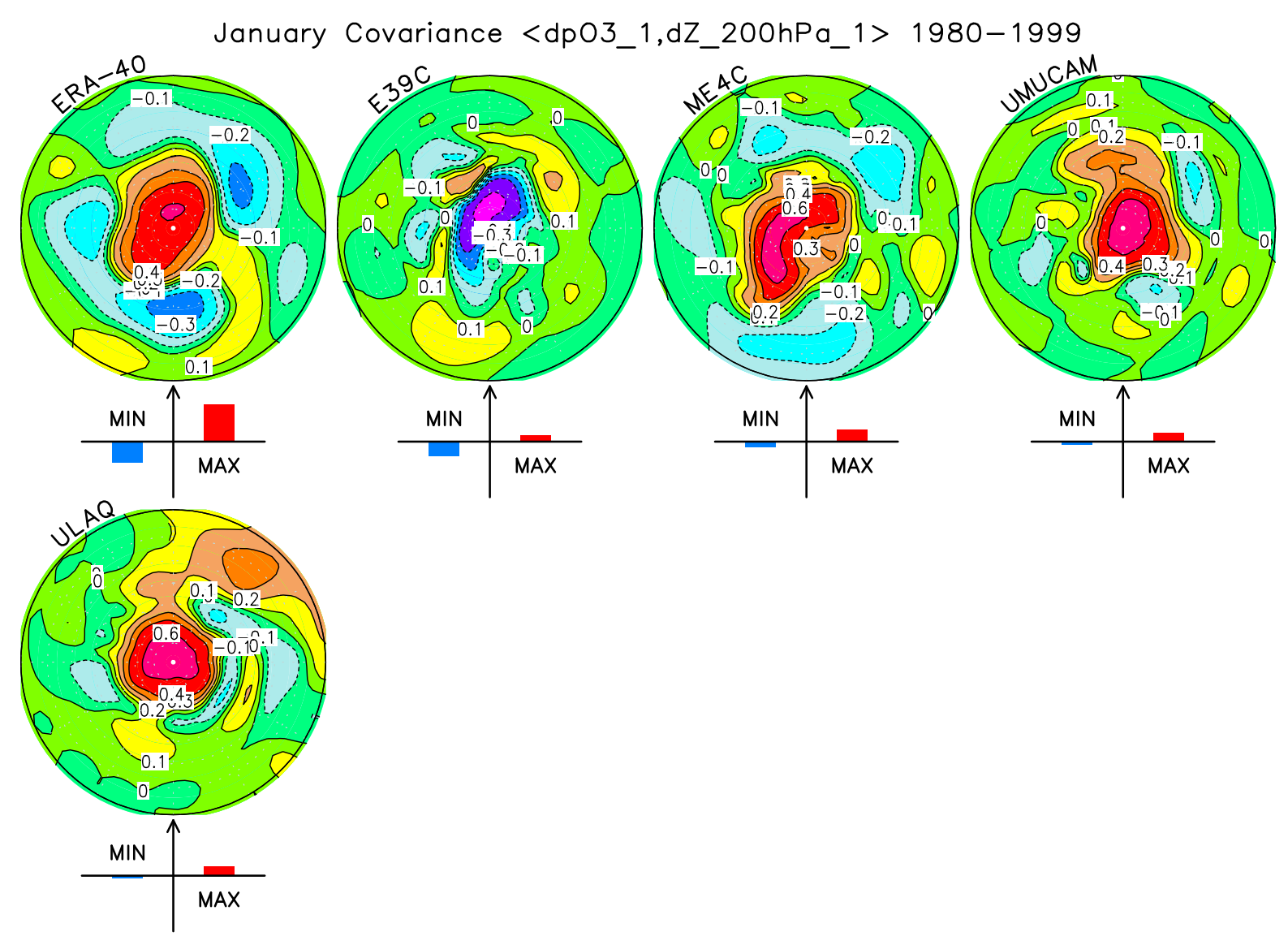

Fig. 11. Covariance of reconstructed geopotential height anomalies at $200 \mathrm{hPa}$ (EOF1) and partial column ozone anomalies (EOF1) for January.

lated with the leading order (EOF1-EOF1) at the two different variables with a continuous drop in amplitude to higher orders. This behaviour is not reproduced in the other models. They show generally higher amplitudes for higher order covariances, within the amplitude range modelled by each model.

A particularly interesting example is the amplitude calculated for EOF2s in geopotential heights and column ozone. Orsolini (2004) describes seesaw fluctuations of column ozone related to North Pacific and North Atlantic surface pressure differences during February and compares those to the AO modulation of column ozone. The Aleutian-Icelandic Index (AII) (as discussed in e.g. Honda and Nakamura (2001)) used in this paper correlates highly with the PNA pattern and the AII regressed column ozone shows more pronounced out-of-phase extremas over both, the North Pacific and North Atlantic, compared to an surface annular mode regressed February column ozone map. The covariance for column ozone (EOF2) and geopotential height anomalies at $200 \mathrm{hPa}$ (EOF2) (Fig. 13) reveal pronounced maxima over the Pacific sector for ERA-40, ME4C and UMUCAM. The pattern is similar to the one revealed by the AII regression on

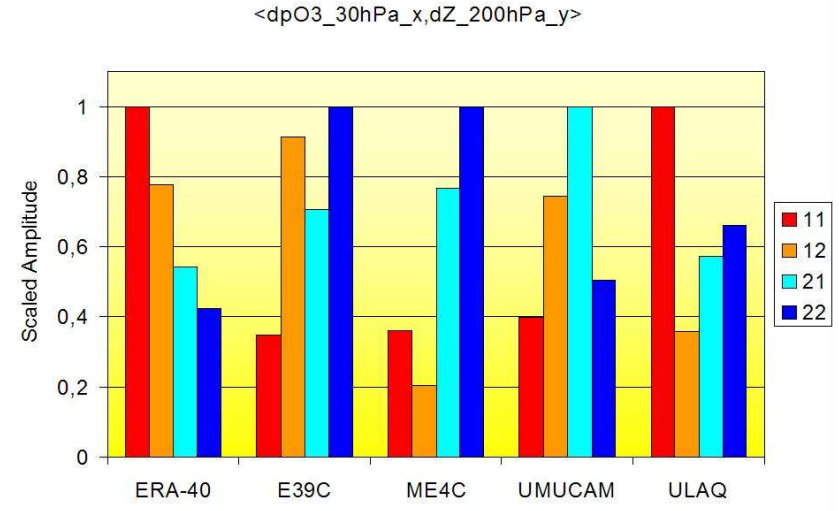

Fig. 12. Amplitudes of ozone/geopotential height covariance patterns scaled with the maximum amplitude found in each model system. 


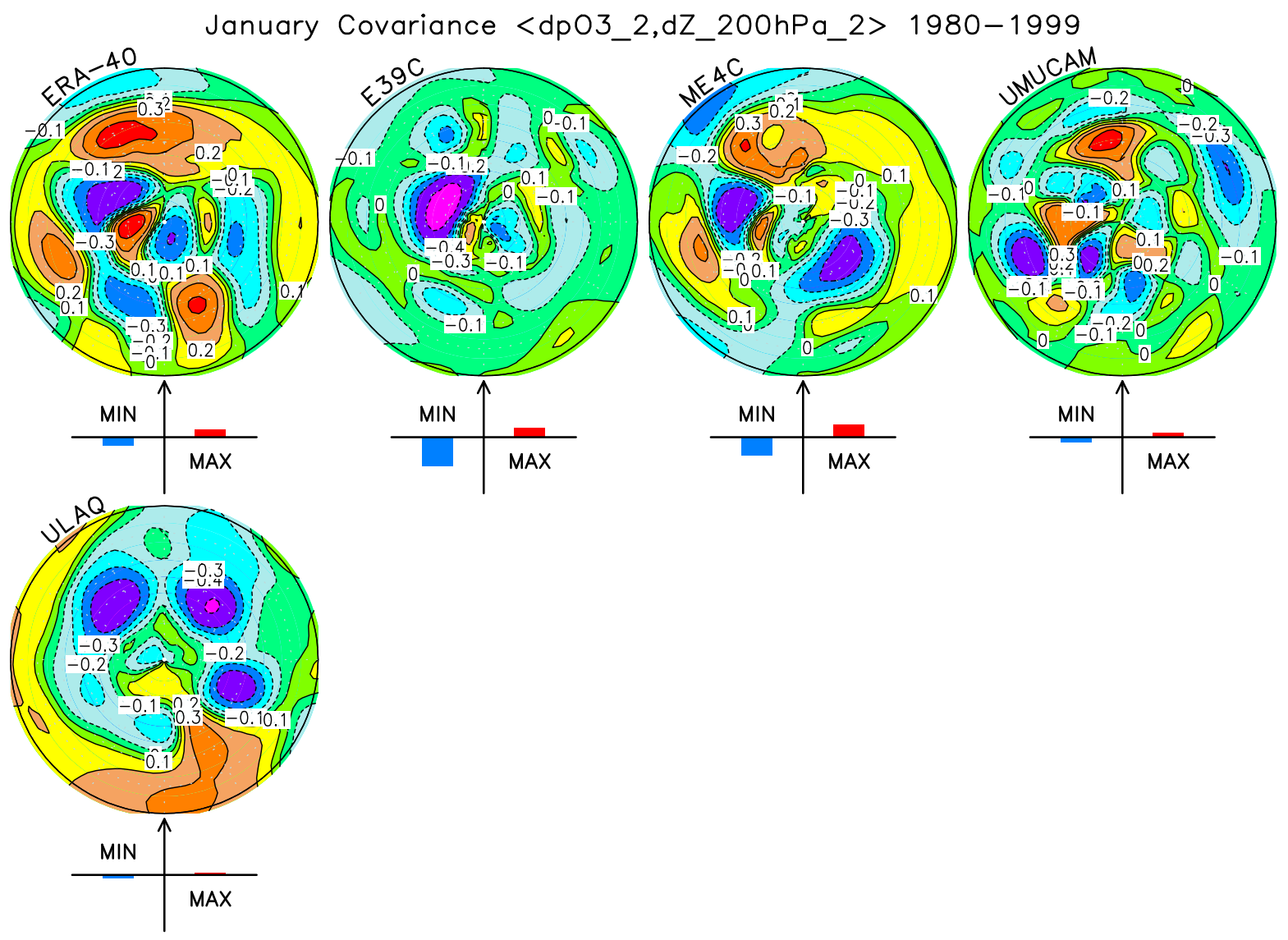

Fig. 13. Covariance of reconstructed geopotential height anomalies at $200 \mathrm{hPa}$ (EOF2) and partial column ozone anomalies (EOF2) for January.

column ozone for February (Fig. 5 in Orsolini, 2004). The hemispheric agreement between ERA- 40 and ME4C is best, with more small scale structures visible in UMUCAM Even though E39C has a strong signal the extrema are very close to the pole.

The agreement between CCMs with higher horizontal resolution and ERA-40 data is generally good with respect to the overall pattern, even though there are differences in the relative amplitude of the pattern. The model with the lowest upper lid (E39C) displays a preference for the tropospheric EOF2 being more important compared to ME4C and the UMUCAM model. The ULAQ model agrees well for EOF1EOF1 covariances only and shows, in all cases discussed, the weakest amplitude.

The agreement between CCMs with higher horizontal resolution and ERA-40 data is generally good with respect to the overall pattern, even though there are differences in the relative amplitude of the pattern. The model with the lowest upper lid (E39C) displays a preference for the tropospheric EOF2 being more important compared to ME4C and the UMUCAM model. The ULAQ model agrees well for EOF1EOF1 covariances only and shows, in all cases discussed, the weakest amplitude.

\subsection{Vertical polar temperature profiles}

The importance of the vertical descretization in numerical models of the atmosphere has been discussed extensively (e.g. Simmons and Burridge, 1981. In addition, care has to be taken in selecting the right upper boundary condition (including the spacing of the vertical levels and damping mechanisms) to avoid arbitrary reflection of vertically propagating waves. A simple measure for buoyancy controlled waves is the vertical temperature gradient. It provides some insight in how the vertical layering of the model and the chosen upper boundary condition affect the (thermo-)dynamic structure of a model.

Figure 14 shows January polar mean temperature profiles averaged over $70^{\circ} \mathrm{N}$ northward (left) and corresponding vertical temperature gradients (right) for all four CCMs and ERA-40. Note that the area for the averaging is somehow arbitrarily chosen. The following discussion will only attempt to illustrate the points made above in terms of two very basic quantities: an averaged temperature profile and the associated vertical gradient. There are three points to note:

- The UMUCAM model is the coldest in the middle stratosphere and $\mathrm{E} 39 \mathrm{C}$ and $\mathrm{ME} 4 \mathrm{C}$ are colder in the 

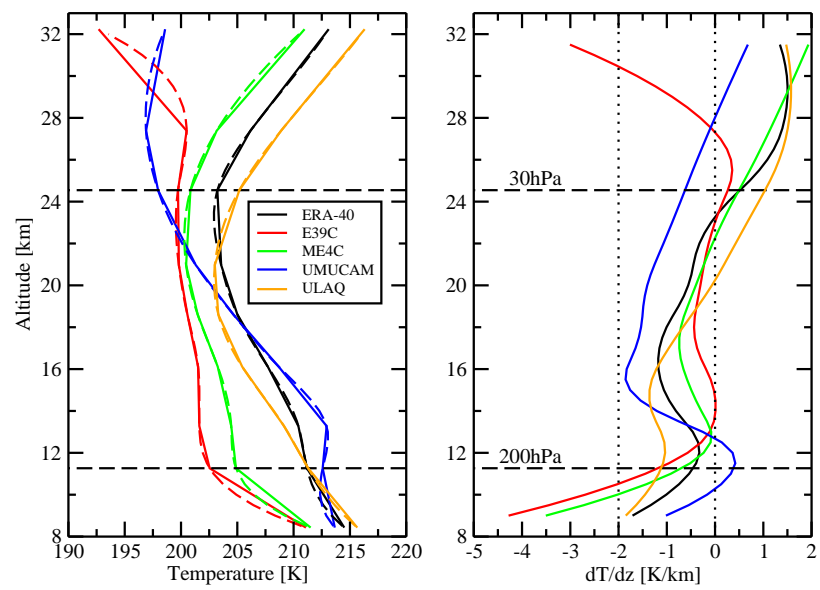

Fig. 14. Left: January polar mean temperature profiles averaged over 70. N northward. Right: Vertical temperature gradients derived from interpolated temperature profiles (dashed lines, left).

lowermost stratosphere, where ULAQ and UMUCAM are reasonably matched to ERA-40.

- The vertical temperature gradient reverses in E39C above $26 \mathrm{~km}$. This feature is quite certainly related to the lower upper boundary and seems to be consistent with the stronger impact of tropospheric lower wavenumbers/higher order EOFs as revealed by the above analysis.

- Even though the ULAQ model matches the temperatures in the stratosphere well compared to ERA-40, it has a less pronounced tropospheric local maximum in the temperature gradient.

Even though this is a very simple diagnostic and not independent from the flowfield and the resolution of the models, the results are consistent with the overall behaviour of the models as shown by the covariance analysis. It is encouraging to note that all troposphere resolving CCMs with a stratosphere do show some similarities in the coupled interannual variability of column ozone and geopotential heights.

\section{Summary, conclusions and outlook}

We applied a statistical analysis framework to analyse some aspects of the combined interannual variability of northern hemisphere column ozone and meteorology during midwinter (January) in four CCMs and in ERA-40.

We confirmed a general picture of the vertical coherence of the models during January on the northern hemisphere and a conceptual interpretation for a simple link between column ozone and meteorology (as represented by $200 \mathrm{hPa}$ geopotential height anomalies) during January, discussing the combined effect of meridional transport towards high latitudes, vortex strength and variations in tropause height in middle latitudes.

The statistical significance of many of our results is low, not withstanding the fact that some quantities will show significant differences on a decadal timescales in idealised model simulations (Braesicke and Pyle, 2003). Nevertheless, it is encouraging that understanding, based on physical processes, is consistent with many aspects of the correlation/covariance structures which we diagnose.

For the spatial patterns of the geopotential height EOF1 at different pressure levels (the annular mode) we find good agreement between the models with variable surface pressure (E39C, ME4C and UMUCAM) and the re-analysis data (ERA-40). The model with a fixed surface pressure (ULAQ) has some problems with the tropospheric annular mode and the NAO related asymmetries, but does perform reasonably well in the lower stratosphere. Note that a recent study by Stenchikov et al. (2006) analysed the Arctic Oscillation (AO) response to volcanic eruptions as simulated by IPPC AR4 models and found a general underestimation of the $\mathrm{AO}$ variability, which is in general agreement with the low CCM amplitudes of the EOF1-EOF1 covariances between 30 and $200 \mathrm{hPa}$ (not shown).

Most models in this study display a reasonable amount of vertical coupling (e.g. a significant amplitude in the covariance) in their geopotential height anomalies, with the ULAQ model showing the weakest vertical coherence. E39C seems to prefer a coupling involving higher tropospheric EOFs (EOF1-EOF2 coupling) to reproduce the overall positive correlation in polar latitudes between tropospheric and stratospheric polar height anomalies, whereas ME4C and the UMUCAM model both show a clear EOF1-EOF1 coupling.

For the covariances between column ozone and geopotential height anomalies at $200 \mathrm{hPa}$ we find good agreement between the CCMs with higher horizontal resolution and ERA40 data with respect to the overall pattern, even though there are differences in the relative amplitudes of the pattern. The model with the lowest upper lid (E39C) displays again a preference for the tropospheric EOF2 being more important compared to ME4C and the UMUCAM model. The ULAQ model agrees well for the EOF1-EOF1 covariance only and shows in all cases discussed the weakest amplitude.

The PNA pattern emerges as a useful qualitative benchmark for the model performance. Models with higher horizontal resolution and high upper boundary (ME4C and UMUCAM) show good agreement with the PNA tripole derived from ERA-40 data, including the column ozone modulation over the Pacific sector. The model with lowest horizontal resolution does not show a classic PNA pattern (ULAQ), and the model with the lowest upper boundary (E39C) does not capture the PNA related column ozone variations over the Pacific sector.

The above has implications for the use of CCMs in climate predictions. The findings presented here should be kept in mind when analysing model simulations for the near and 
far future. As long as we are sure that the modes of variability stay similar under climate change (as prescribed by chosen boundary conditions) the troposphere resolving models should perform well (the assumption about similar modes is only save for the near future, assuming that we are not to close to a critical threshold). Note that other model assumptions may need adjusting, e.g. the parameterised ozone chemistry in UMUCAM (depending on the application). Simpler models need to restrict their interpretation of future climate to sensitivity studies.

Future work will also focus on the spring season, analysing the ability of models to simulate the dynamical control of ozone during and after the stratospheric vortex break-up in middle latitudes on the northern hemisphere (e.g. Orsolini and Doblas-Reyes, 2003) and the same methodology can be used to assess climate change integrations.

Acknowledgements. The authors would like to acknowledge the support of the EU integrated project SCOUT-O3. In addition, P. Braesicke and J. A. Pyle would like to thank NERC for support through NCAS. We would like to thank two anonymous reviewers for their supportive and constructive comments.

Edited by: W. T. Sturges

\section{References}

Ambaum, M. H. P., Hoskins, B. J., and Stephenson, D. B.: Arctic oscillation or North Atlantic oscillation?, J. Climate, 14(16), 3495-3507, 2001.

Baldwin, M. P.: Annular modes in global daily surface pressure, Geophys. Res. Lett., 28, 4115-4118, 2001.

Baldwin, M. P. and Dunkerton, T. J.: Propagation of the Arctic Oscillation from the stratosphere to the troposphere, J. Geophys. Res.-Atmos., 104(D24), 30 937-30 946, 1999.

Braesicke, P., Jrrar, A., Hadjinicolaou, P., and Pyle, J. A.: Variability of total ozone due to the $\mathrm{NAO}$ as represented in two different model systems, Met. Z., 12, 203-208, 2003.

Braesicke, P. and Pyle, J. A.: Changing ozone and changing circulation in northern mid-latitudes: Possible feedbacks?, Geophys. Res. Lett., 30, 1059, doi:10.1029/2002GL015973, 2003.

Braesicke, P. and Pyle, J. A.: Sensitivity of dynamics and ozone to different representations of SSTs in the Unified Model, Q. J. R. Meteorol. Soc., 130, 2033-2045, 2004.

Cariolle, D. and Déqué, M.: Southern hemisphere medium-scale waves and total ozone disturbances in a spectral general circulation model, J. Geophys. Res., 91, 10 825-10 846, 1986.

Christiansen, B.: On the physical nature of the Arctic Oscillation, Geophys. Res. Lett., 29(16), doi:10.1029/2002GL015208, 2002.

Cohen, J. and Saito K.: A test for annular modes, J. Climate, 15(17), 2537-2546, 2002.

Dameris, M., Grewe, V., Ponater, M., Deckert, R., Eyring, V., Mager, F., Matthes, S., Schnadt, C., Stenke, A., Steil, B., Brühl, C., and Giorgetta, M. A.: Long-term changes and variability in a transient simulation with a chemistry-climate model employing realistic forcing, Atmos. Chem. Phys., 5, 2121-2145, 2005, http://www.atmos-chem-phys.net/5/2121/2005/.
Dameris, M., Matthes, S., Deckert, R., Grewe, V., and Ponater, M.: Solar cycle effect delays onset of ozone recovery, Geophys. Res. Lett., 33, L03806, doi:10.1029/2005GL024741, 2006.

Dethof, A. and Holm, E. V.: Ozone assimilation in the ERA-40 reanalysis project, Q. J. Roy. Meteorol. Soc., 130, 2851-2872, 2004.

Dobson, G. M. B.: Observations of the amount of ozone in the Earth's atmosphere and its relations to other geophysical conditions, Proc. Soc. London Ser. A, 129, 411-433, 1930.

Eyring, V., Harris, N. R. P., Rex, M., et al.: A strategy for processoriented validation of coupled chemistry-climate models, Bull. Am. Meteorol. Soc., 86(8), 1117-1133, 2005.

Eyring, V., Butchart, N., Waugh, D. W., et al.: Assessment of temperature, trace species, and ozone in chemistry-climate model simulations of the recent past, J. Geophys. Res., D22308, doi:10.1029/2006JD007327, 2006.

Graf, H.F., and K. Walter: Polar vortex controls coupling of North Atlantic Ocean and Atmosphere, Geophys. Res. Lett., 32(1), L01704, doi:10.1029/2004GL020664, 2005.

Honda, M., and Nakamura, H.: Interannual seesaw between the Aleutian and Icelandic lows. Part II: Its significance in the interannual variability over the wintertime Northern Hemisphere, Journal of Climate, 14(24), 4512-4529, 2001.

Kodera, K. and Kuroda, Y.: Regional and hemispheric circulation patterns in the northern hemisphere winter, or the NAO and the AO, Geophys. Res. Lett., 30, 1934, doi:10.1029/2003GL017290, 2003.

Manzini, E., Steil, B., Brühl, C., Giorgetta, M. A., and Krüger, K.: A new interactive chemistry-climate model. 2. Sensitivity of the middle atmosphere to ozone depletion and increase in greenhouse gases: Implications for recent stratospheric cooling, J. Geophys. Res., 108, 4429, doi:10.1029/2002JD002977, 2003.

Orsolini, Y.J.: Seesaw fluctuations in ozone between the North Pacific and North Atlantic, J. Met. Soc. Japan, 82(3), 941-949, 2004.

Orsolini, Y. J., Stephenson, D. B., and Doblas-Reyes, F. J.: Storm track signature in total ozone during northern hemisphere winter, Geophys. Res. Lett., 25, 2413-2416, 1998.

Orsolini, Y. J. and Doblas-Reyes, F. J.: Ozone signatures of climate patterns over the Euro-Atlantic sector in the spring, Q. J. R. Meteorol. Soc., 129, 3251-3263, 2003.

Perlwitz, J. and Graf, H. F.: The statistical connection between tropospheric and stratospheric circulation of the northernhemisphere in winter, J. Climate, 8(10), 2281-2295, 1995.

Perlwitz, J., Graf, H. F., and Voss, R.: The leading variability mode of the coupled troposphere-stratosphere winter circulation in different climate regimes, J. Geophys. Res.-Atmos. 105(D5), 69156926, 2000.

Pitari, G., Mancini, E., Rizi, V., and Shindell, D. T.: Impact of future climate and emission changes on stratospheric aerosols and ozone, J. Atmos. Sci., 59, 414-440, 2002.

Pyle, J. A., Braesicke, P., and Zeng, G.: Dynamical variability in the modelling of chemistry-climate interactions - Faraday Discuss., 130, 27-39, doi:10.1039/b417947c, 2005.

Randel, W., Udelhofen, P., Fleming, E., et al.: The SPARC intercomparison of middle-atmosphere climatologies, J. Climate, 17(5), 986-1003, 2004.

Rex M., Salawitch R. J., von der Gathen P., Harris N. R. P., Chipperfield M. P., and Naujokat B.: Arctic ozone loss and climate 
change, Geophys. Res. Lett., 31, L04116, 2004.

Schnadt, C. and Dameris, M.: Relationship between NAO changes and stratospheric ozone recovery in the northern hemisphere in a chemistry-climate model, Geophys. Res. Lett., 30, doi:10.1029/2003GL017006, 2003.

Simmons, A. J. and Buridge, D.M.: An energy and angularmomentum conserving vertical finite-difference scheme and hybrid vertical-coordinate, Monthly Weather Review, 109(4), 758766, 1981.

Steil, B., Brühl, C., Manzini, E., Crutzen, P. J., Lelieveld, J., Rasch, P. J., Roeckner, E., and Krüger, K.: A new interactive chemistry-climate model. 1. Present day climatology and interannual variability of the middle atmosphere using the model and 9 years of HALOE/UARS data, J. Geophys. Res., 108, 4290, doi:10.1029/2002JD002971, 2003.

Steinbrecht, W., Claude, H., Kohler, U., et al.: Correlations between tropopause height and total ozone: Implications for long-term changes, J. Geophys. Res.-Atmos., 103, 19 183-19 192, 1998.

Steinbrecht, W., Haßler, B., Brühl, C., Dameris, M., Giorgetta, M. A., Grewe, V., Manzini, E., Matthes, S., Schnadt, C., Steil, B., and Winkler, P.: Interannual variation patterns of total ozone and lower stratospheric temperature in observations and model simulations, Atmos. Chem. Phys., 6, 349-374, 2006,

http://www.atmos-chem-phys.net/6/349/2006/.

Stenchikov, G., Hamilton, K., Stouffer, R. J., Robock, A., Ramaswamy, V., Santer, B., and Graf, H.-F.: Arctic Oscillation response to volcanic eruptions in IPCC AR4 climate models, J. Geophys. Res.-Atmos., 111(D7), D07107, doi:10.1029/2005JD006286, 2006.
Thompson, D. W. J. and Wallace, J. M.: The Arctic Oscillation signature in the wintertime geopotential height and temperature fields, Geophy Res. Lett., 25, 1297-1300, 1998.

Thompson, D. W. J., Baldwin, M. P., and Wallace, J. M.: Stratospheric connection to Northern Hemisphere wintertime weather: Implications for prediction, J. Climate, 15(12), 1421-1428, 2002.

Uppala, S. M., Kallberg, P. W., Simmons, A. J., et al.: The ERA-40 re-analysis, Q. J. R. Meteorol. Soc., 131, 2961-3012, 2005.

Wallace, J. M. and Gutzler, D. S.: Teleconnections in the geopotential height field during the northern hemisphere winter, Monthly Weather Rev., 109(4), 784-812, 1981.

Wallace, J. M.: North Atlantic Oscillation/annular mode: Two paradigms - one phenomenon, Q. J. R. Meteorol. Soc., 126, 791$805,2000$.

Wallace, J. M. and Thompson, D. W. J.: The Pacific center of action of the northern hemisphere annular mode: Real or artifact?, J. Climate, 15, 1987-1991, 2002.

Walter, K. and Graf, H. F.: The North Atlantic variability structure, storm tracks, and precipitation depending on the polar vortex strength, Atmos. Chem. Phys., 4, 6127-6148, 2005, http://www.atmos-chem-phys.net/4/6127/2005/. 